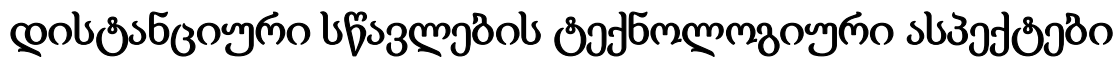 Geogebra Classroom - M ath zsmjamåo
}

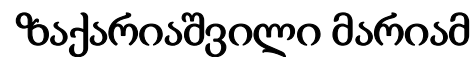

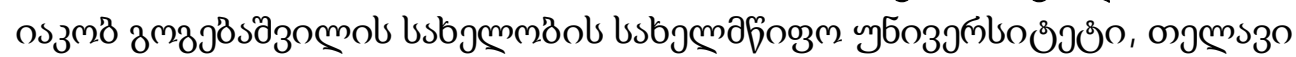 \\ https://doi.org/10.52340/idw.2021.539
}

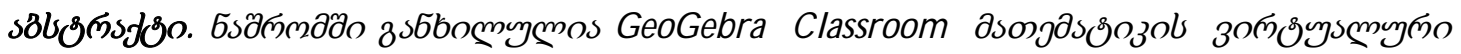

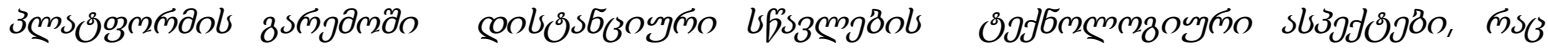

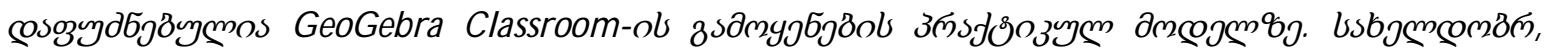

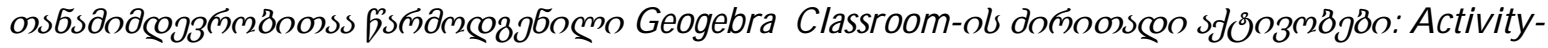

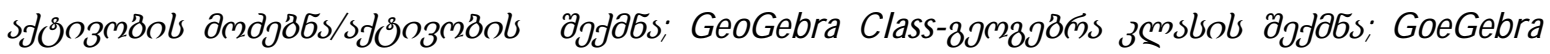

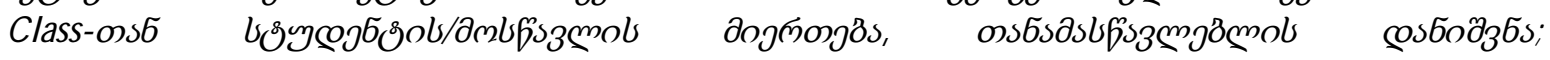

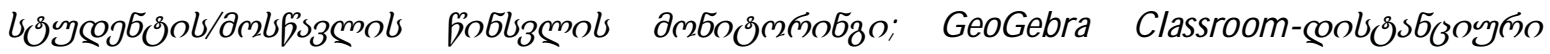

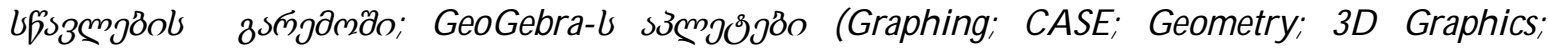
Spreadsheet; Probability; Notes) GeoGebra Classroom - olsonzol; GeoGebra Class-ob J⿹dДбs зэмzjдйl fozбocos6 (GeoGebra-Book).

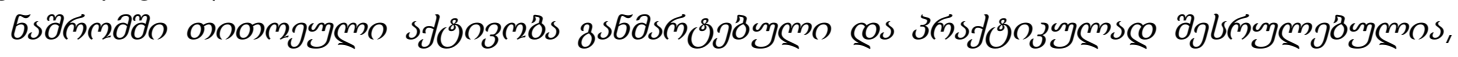

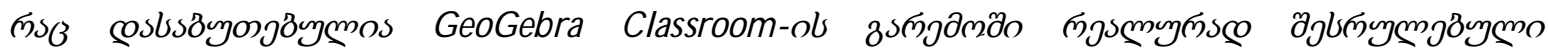

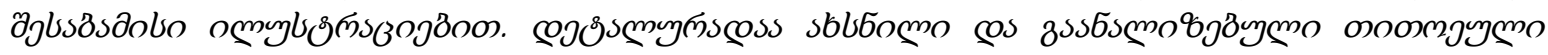

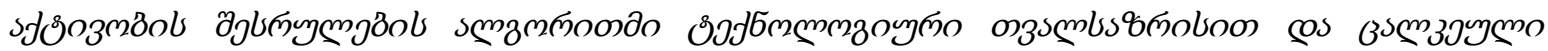

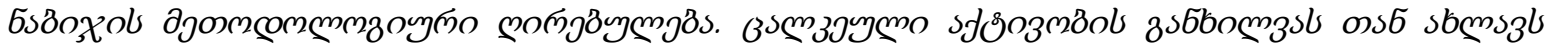

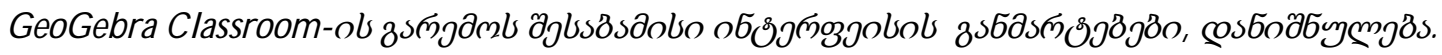

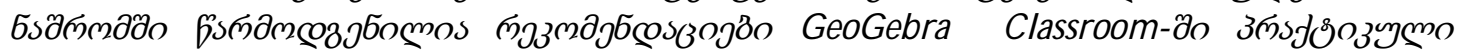

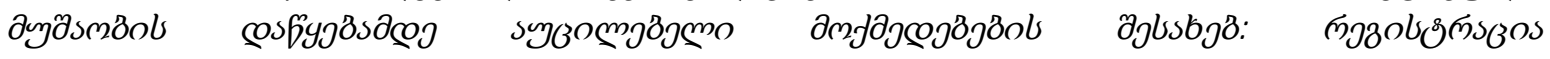

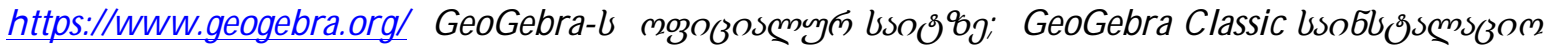

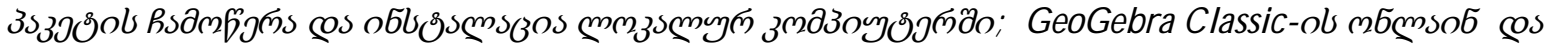

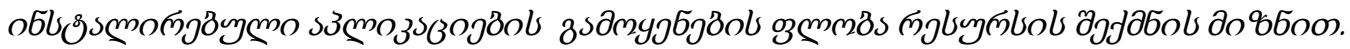

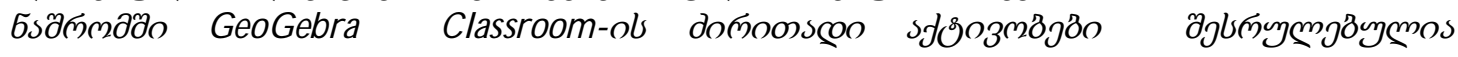

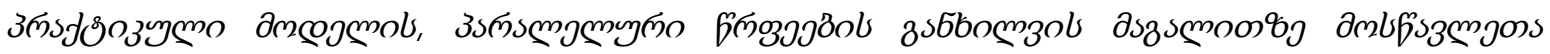

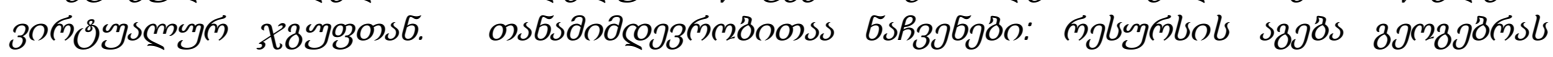

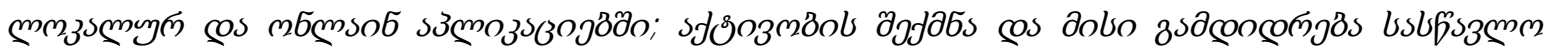

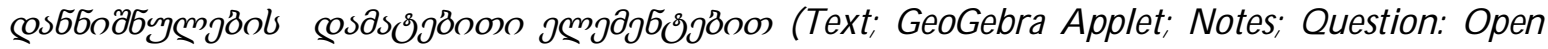
question, Multiple choice; Video; Picture; PDF file Web); з

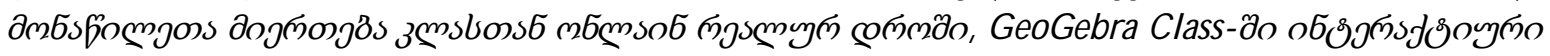

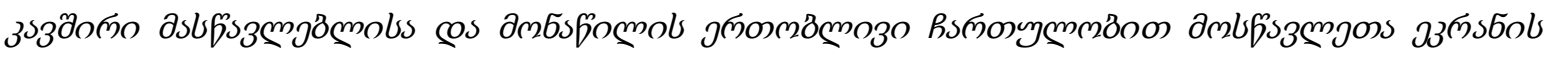

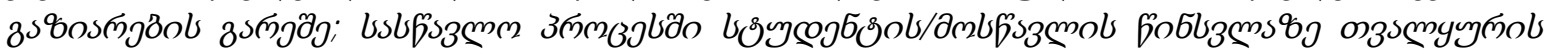

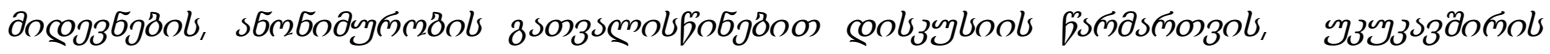
उॅмखjlo.

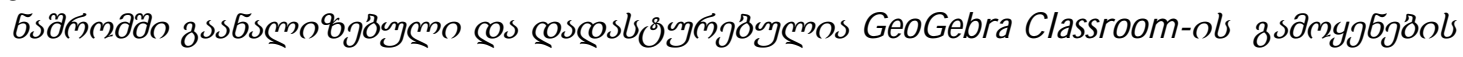

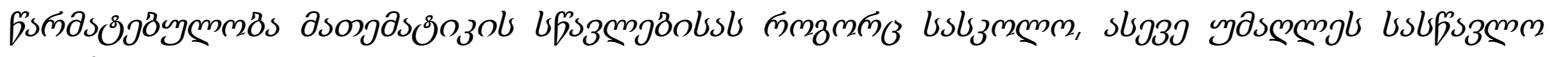

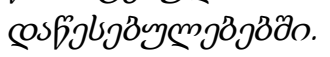

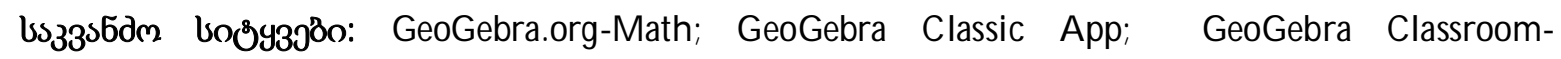

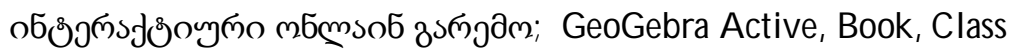




\section{gjlsзsмº}

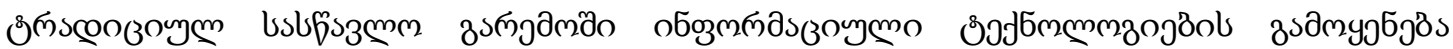

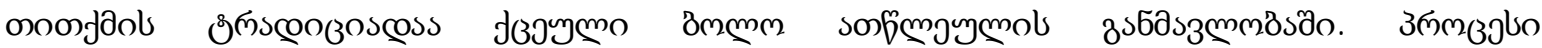

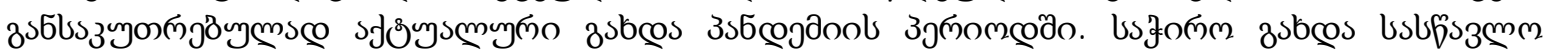

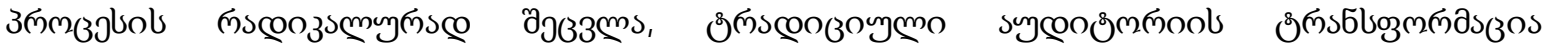

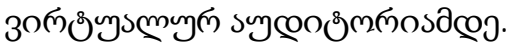

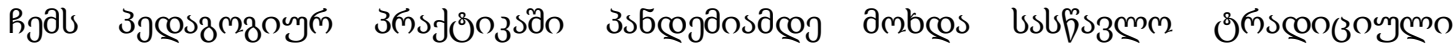

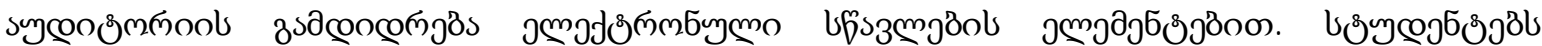

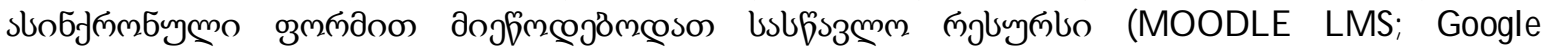

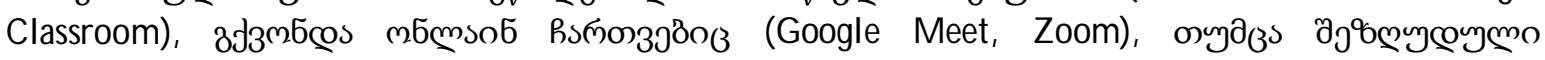

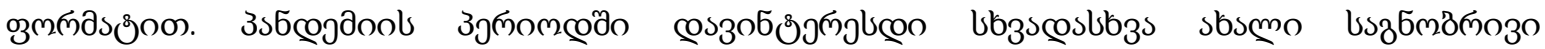

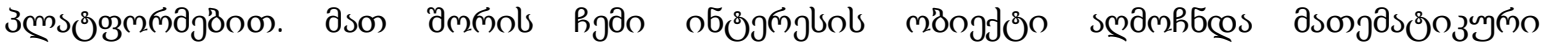

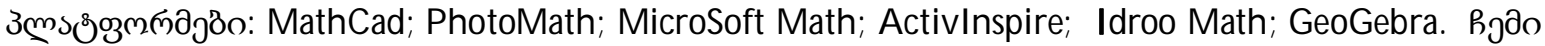

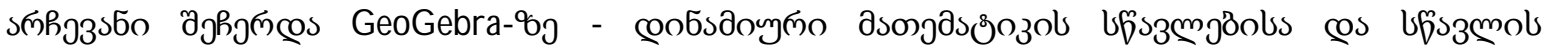

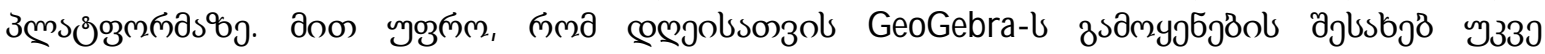

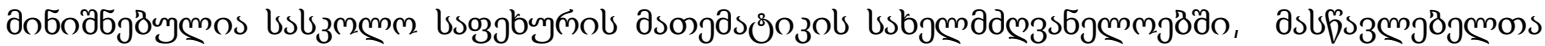

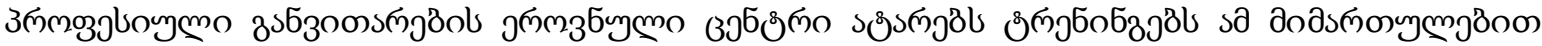

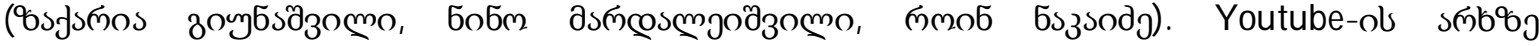

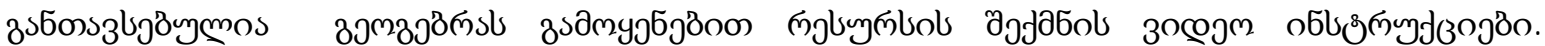

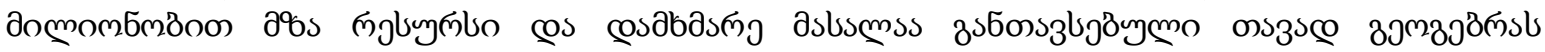

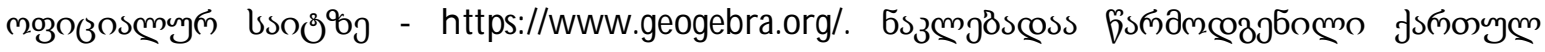

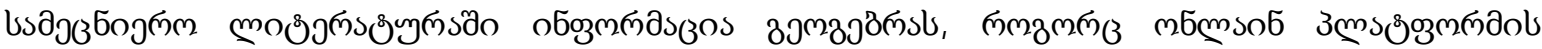

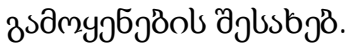

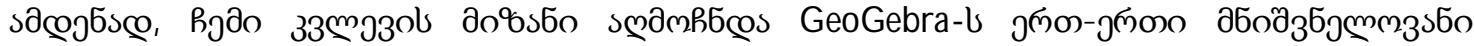

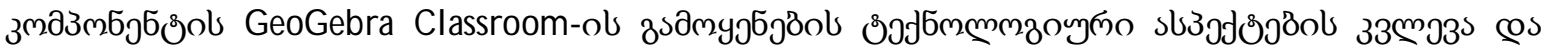

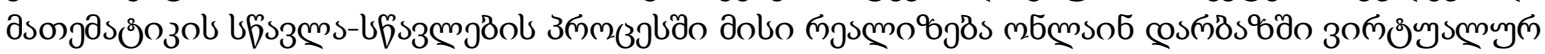

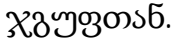

\section{gobssø̆bo}

\section{- ms srnob Geogebra Classroom}

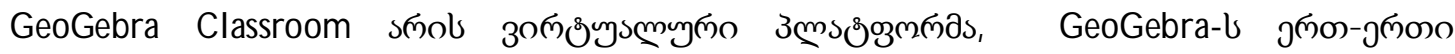

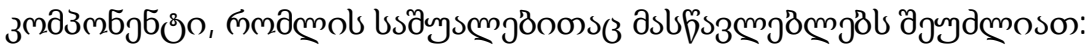

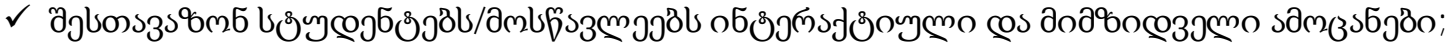

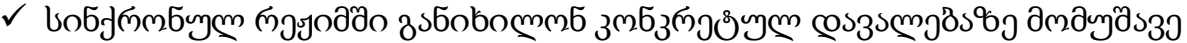

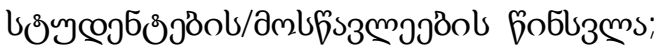

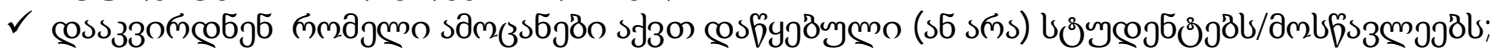

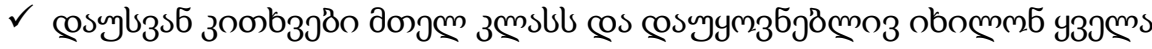

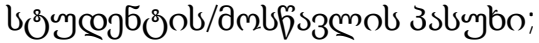

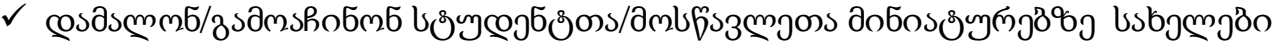

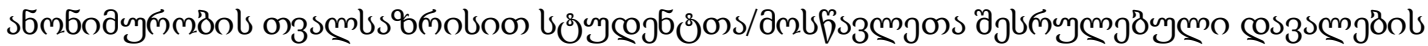
zstbonm3obst;

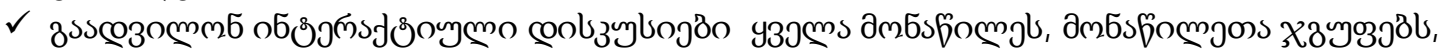

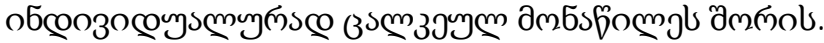

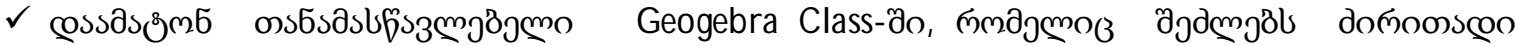

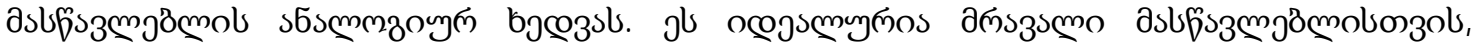

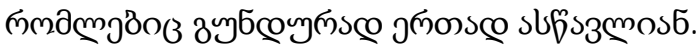

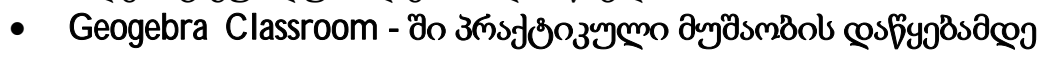

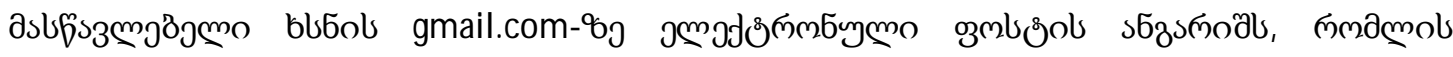
zsamyjбjöon GeoGebra-l mozozosmyn usoơ 


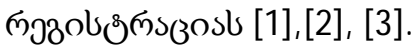

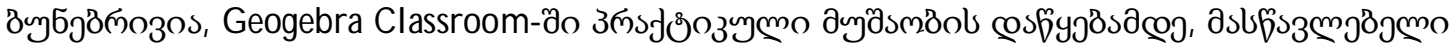

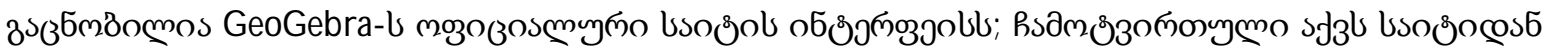

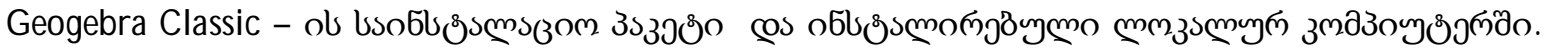

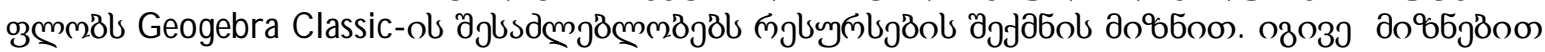

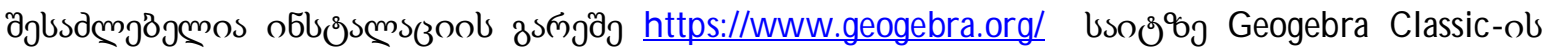
мбмsoб zsamyjбjös. [1] ,[2], [3].

- GeoGebra Classroom-ol domoossco sjoిozmżzon:

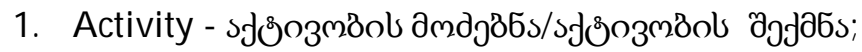

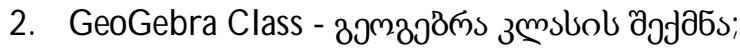

3. GoeGebra Class - osb b

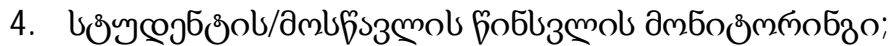

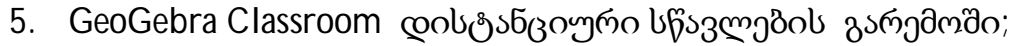

6. GeoGebra -l sзмmozszojöo Geogebra Classroom - obsozzob;

7. GeoGebra Class-ob goja6s fozбocos6

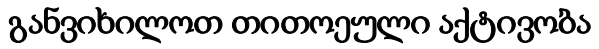

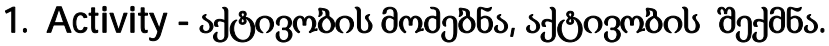

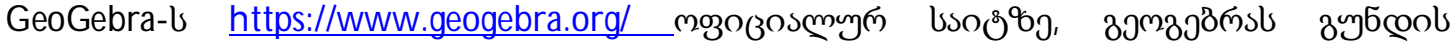

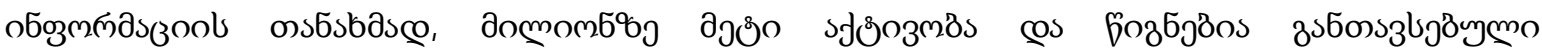

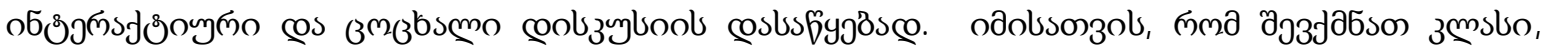

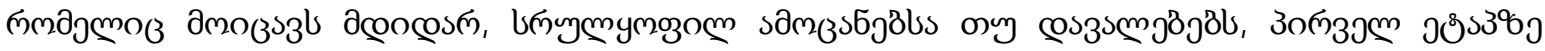

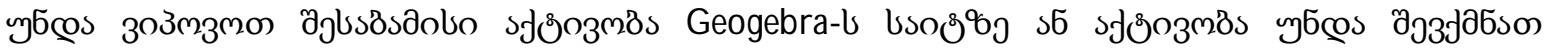

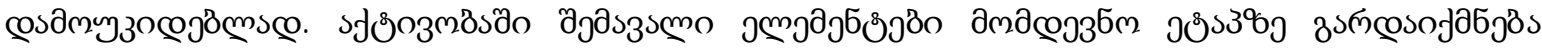

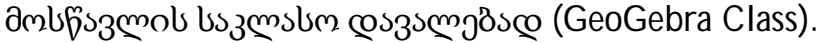

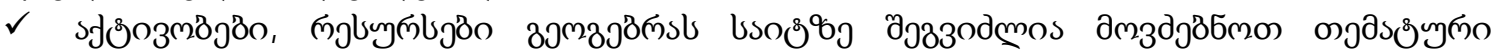
coszmsaols cosbasmjöon https://www.geogebra.org/materials (Math: Arithmetic; Geometry;

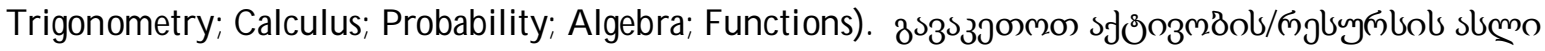

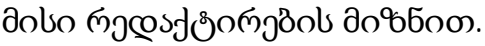

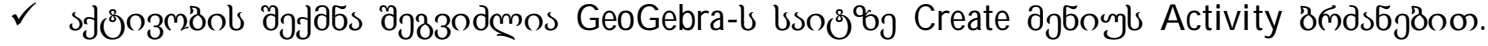

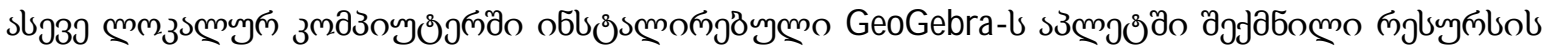

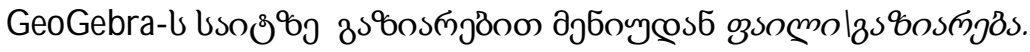

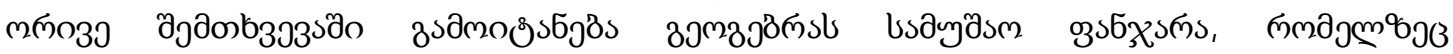

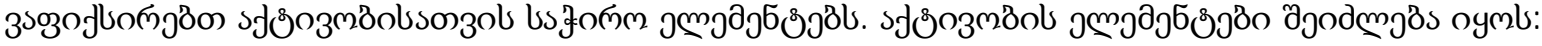

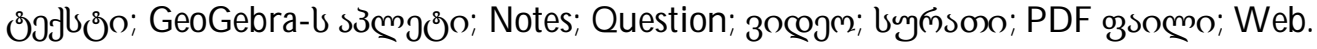

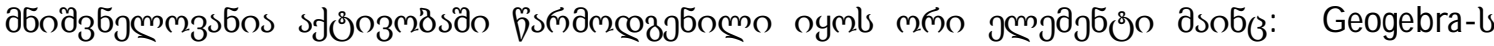

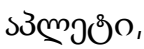

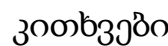

(Questions).

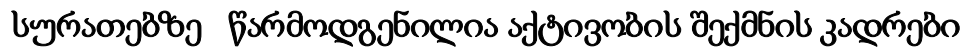

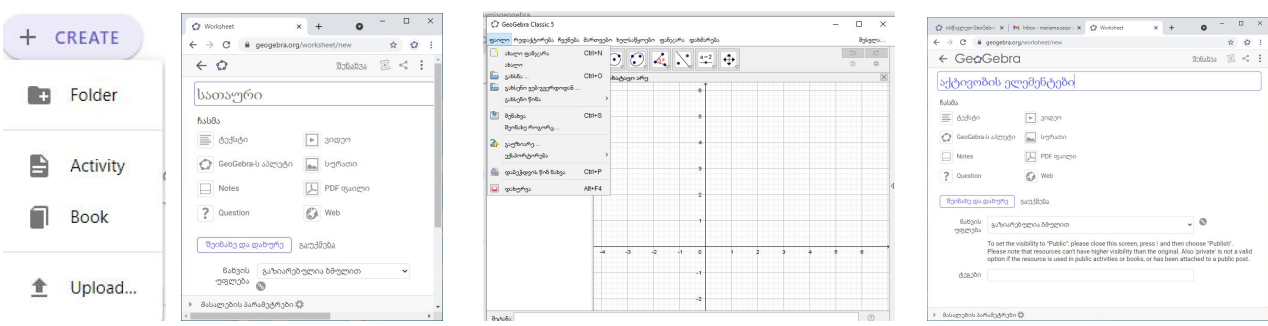

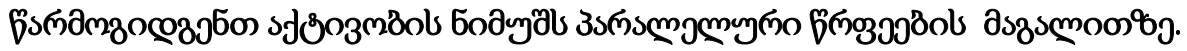

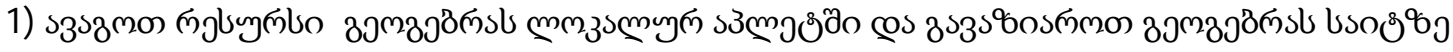

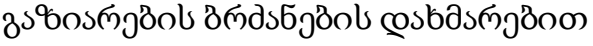

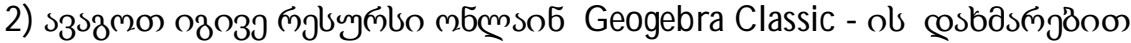

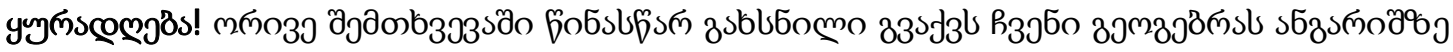




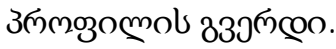
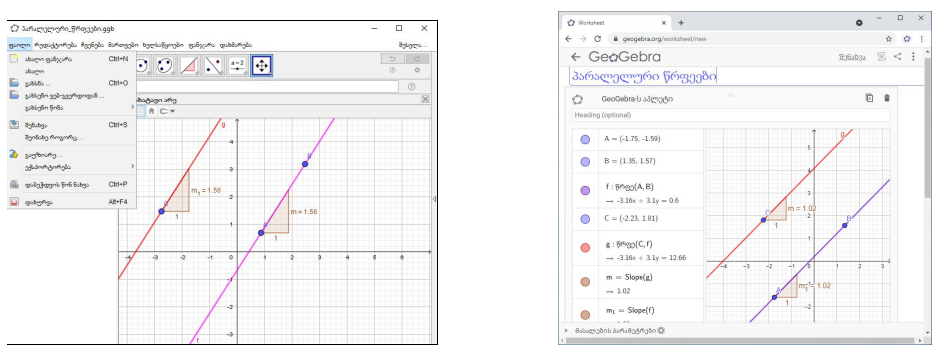

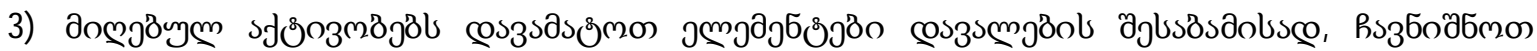

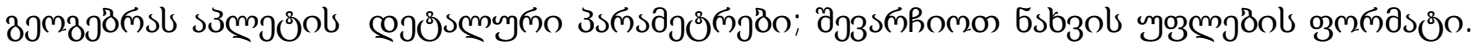

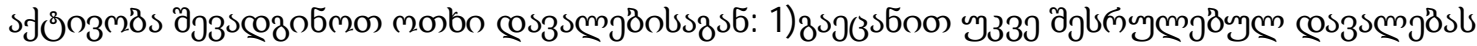

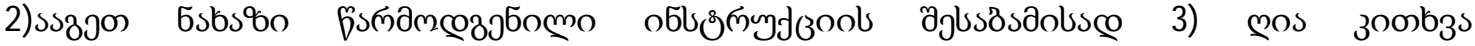

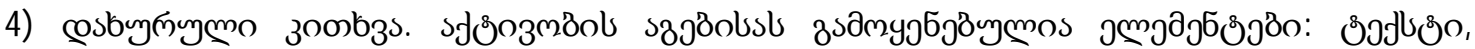
GeoGebra A ppl et: nos cos cosbyñymo zoob $3 j 8 ̈$ o.

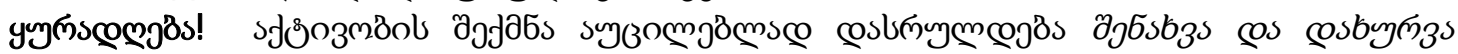

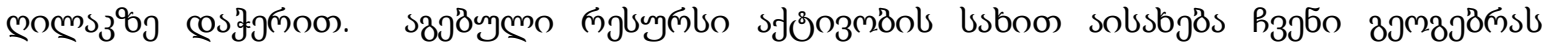

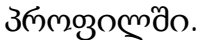

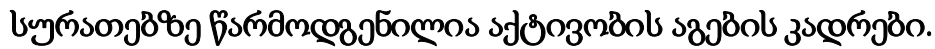
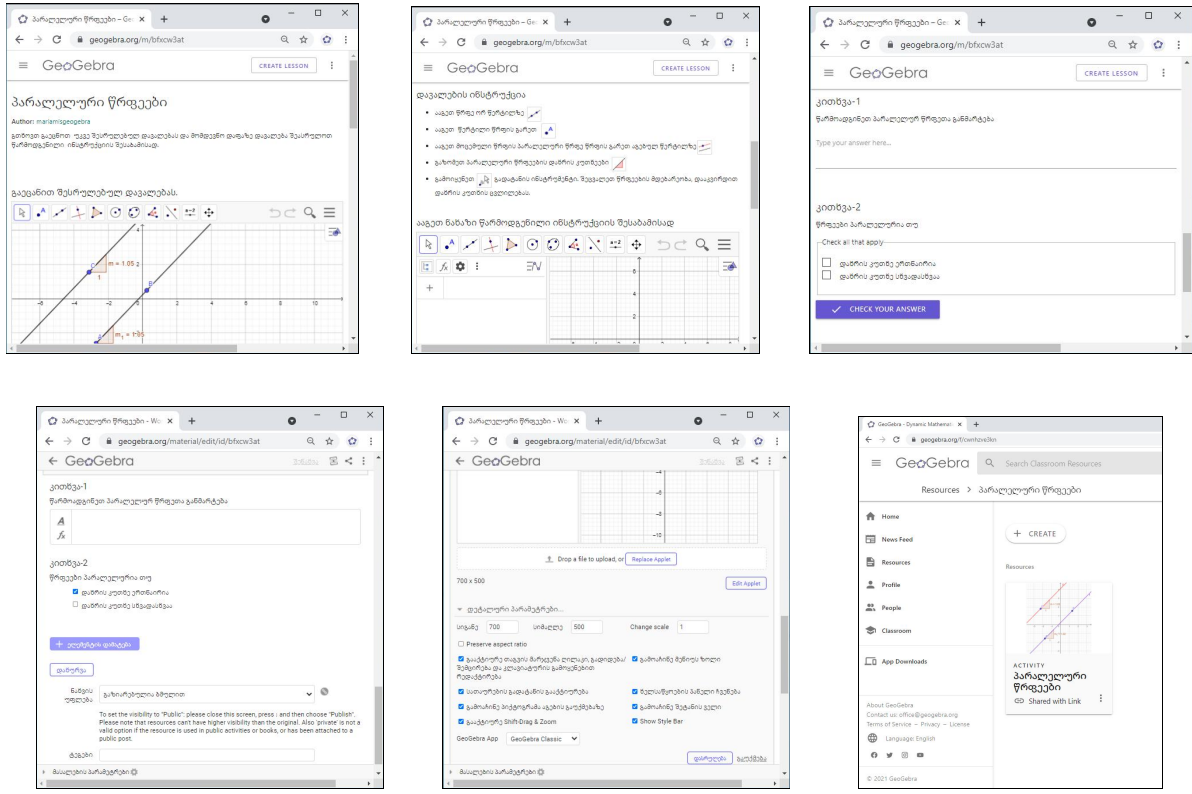

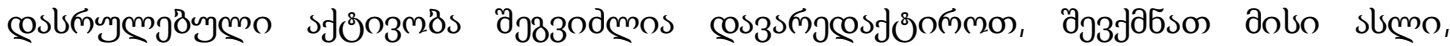

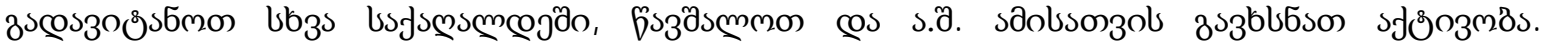

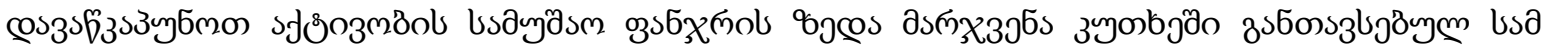

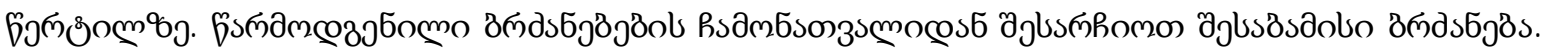

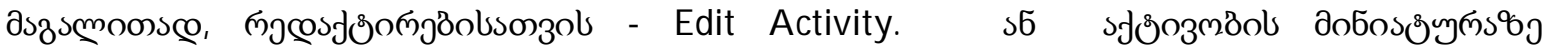

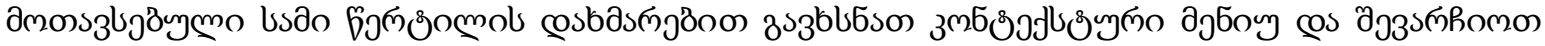

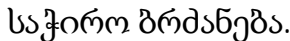

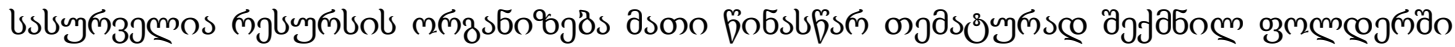

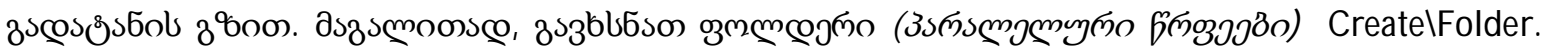

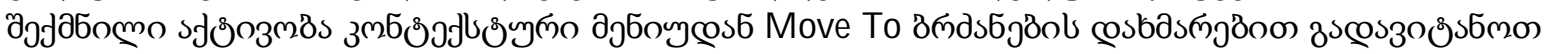

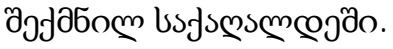

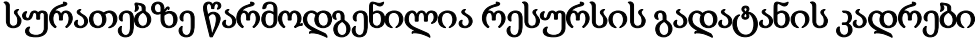



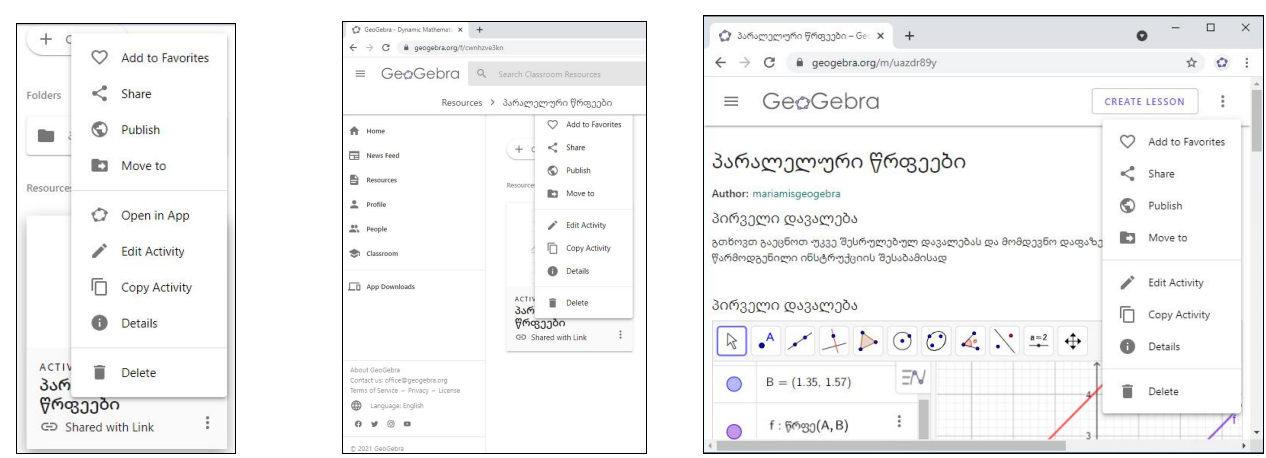

\section{GeoGebra Class - ob gojabs}

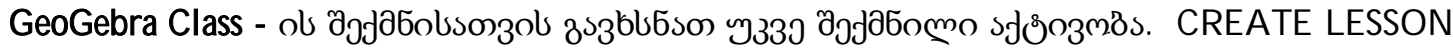

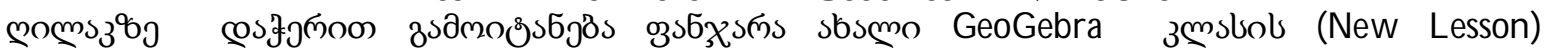

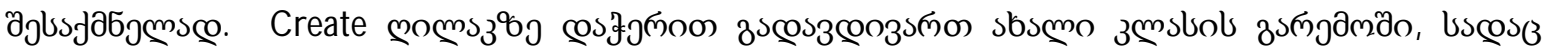

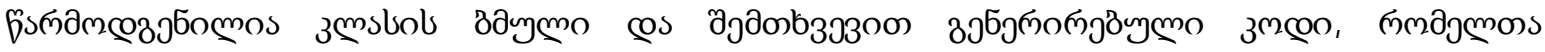

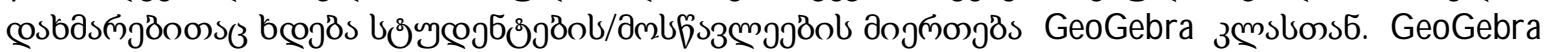

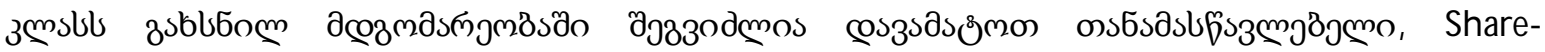

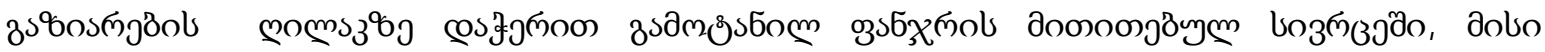

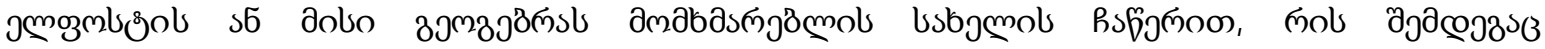

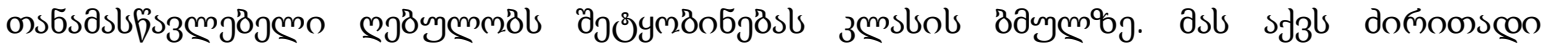

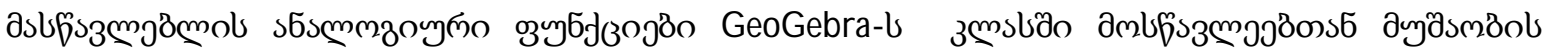

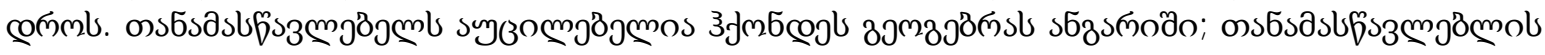

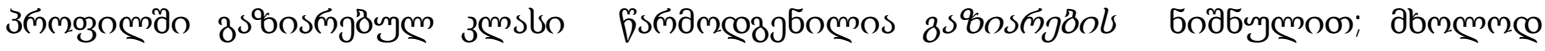

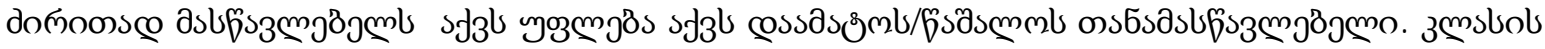

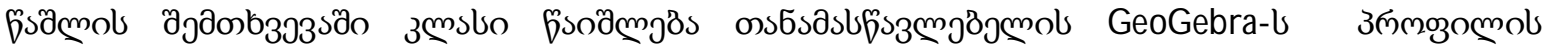

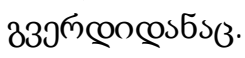

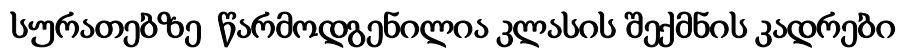

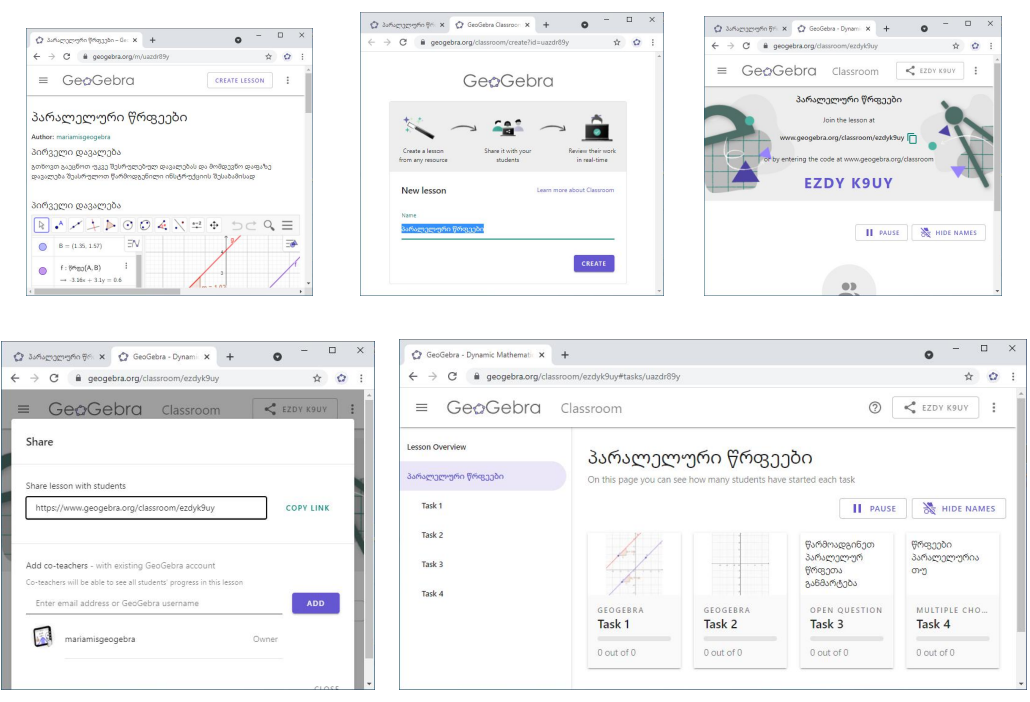

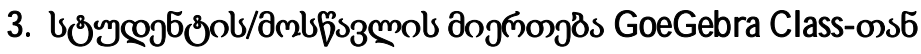

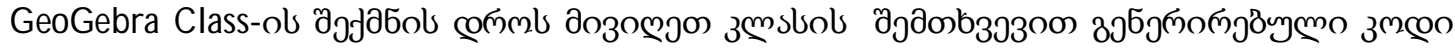
(EZDY K9UY) cos https://www.geogebra.org/classroom/ezdyk9uy

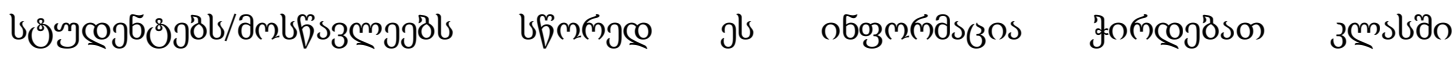




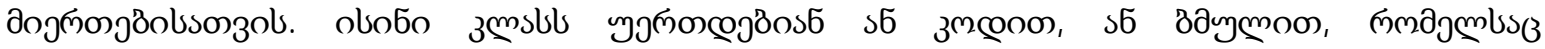

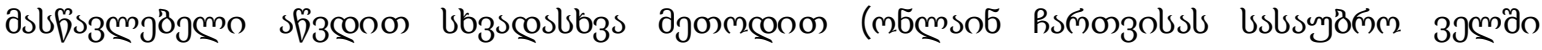

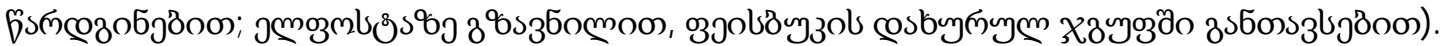

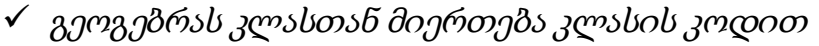

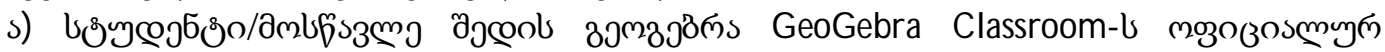
usos\%g https://www.geogebra.org/classroom

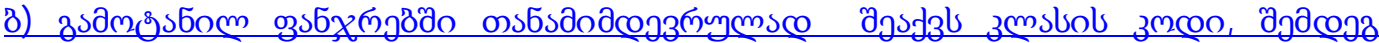
usbjemo

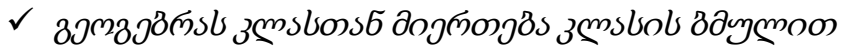

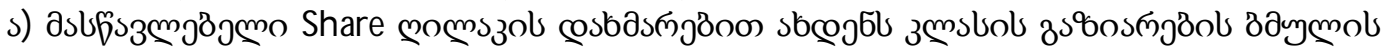

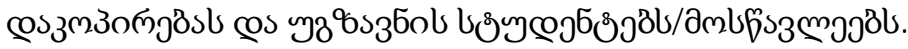

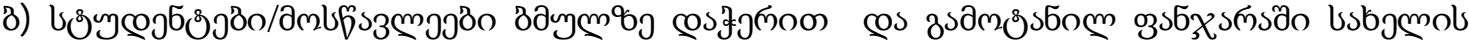

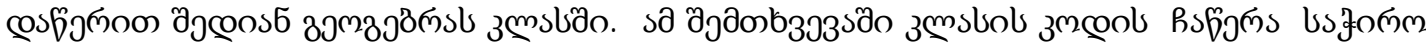
sल smol.

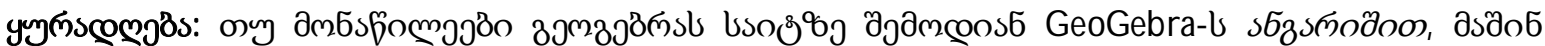

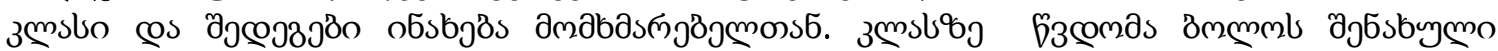

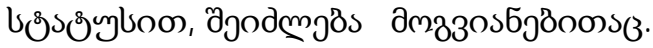

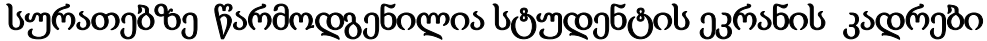

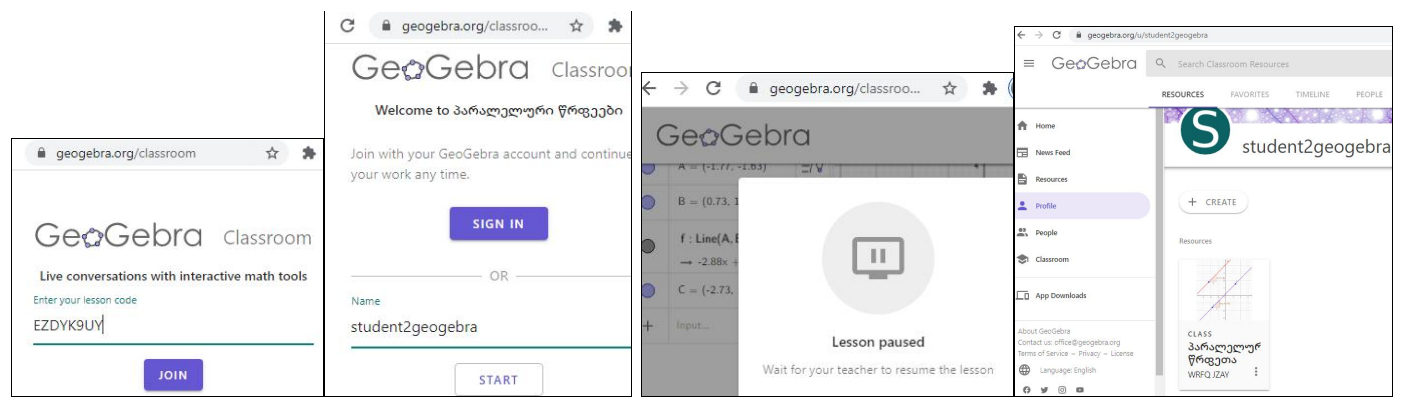

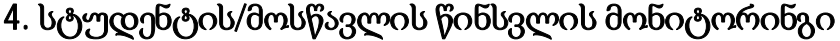

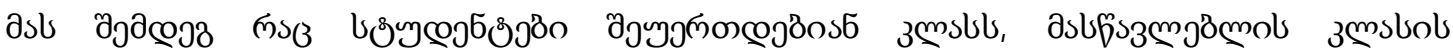

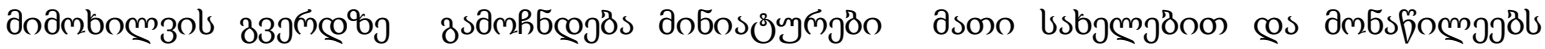

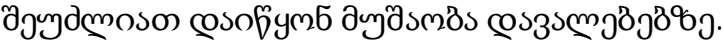

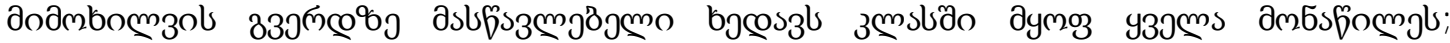

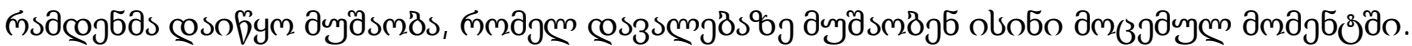

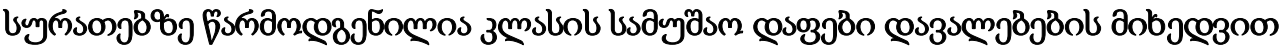
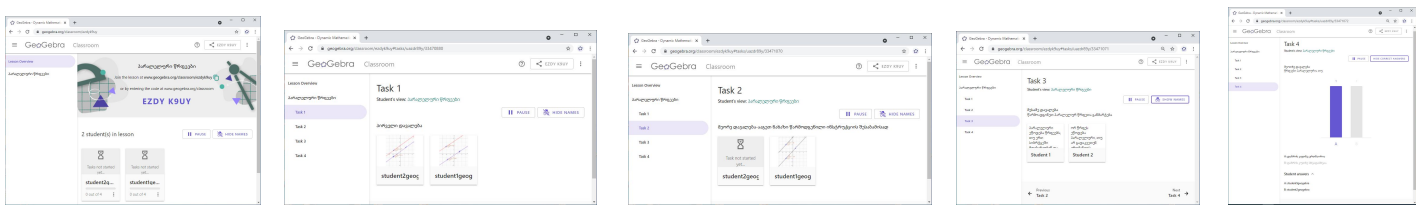

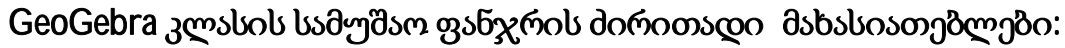

LESSON OVERVIEW - зmslsol дodmbomzs. jl

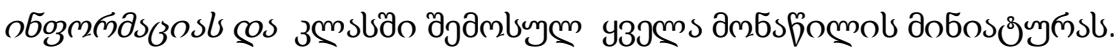

$\checkmark$ з

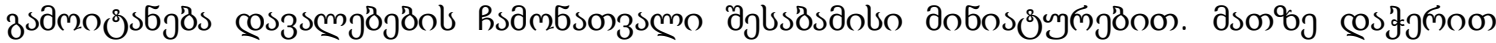

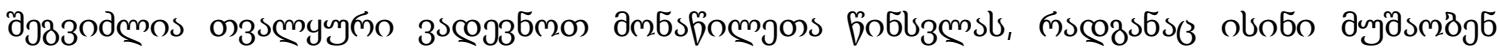

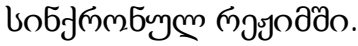




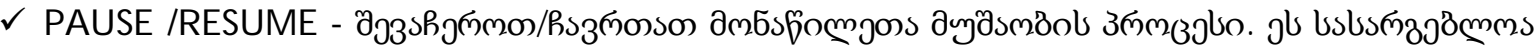

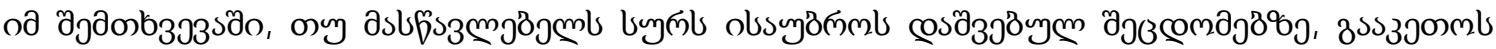

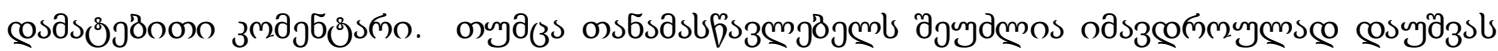
ambsfocmols aỹasmzols asbsbmgßs.

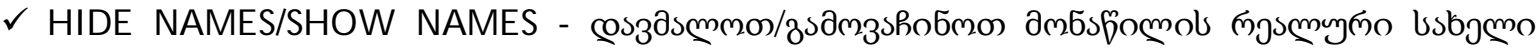

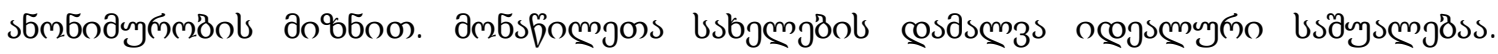

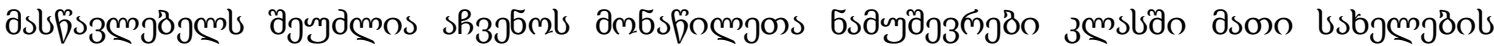

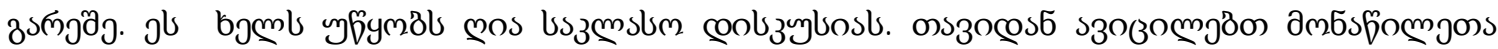

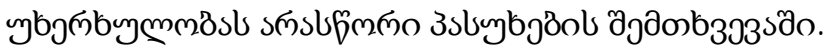

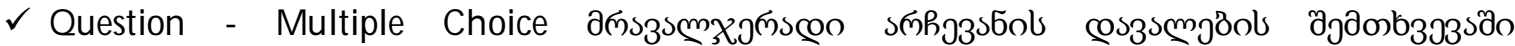

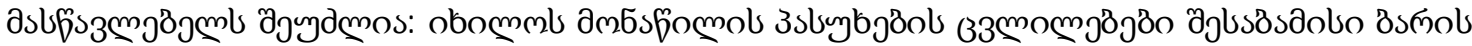

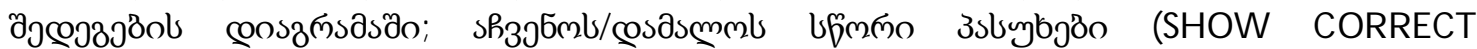

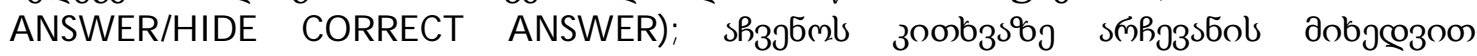
дmбsfom

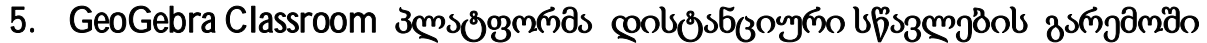

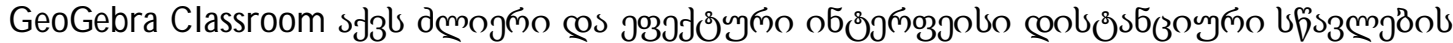

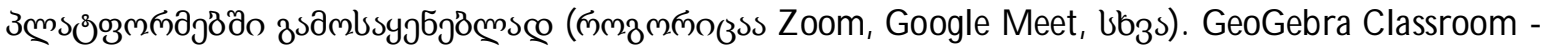

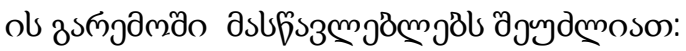

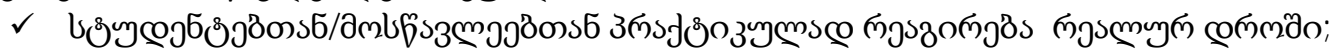

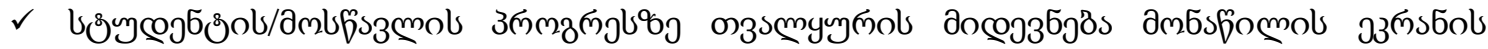

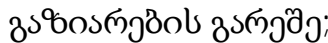

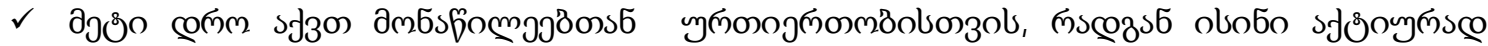

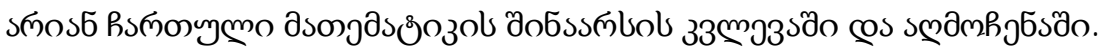

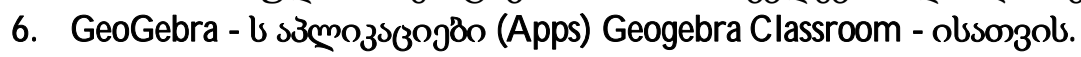

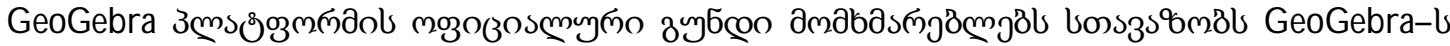

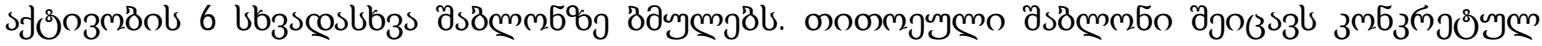

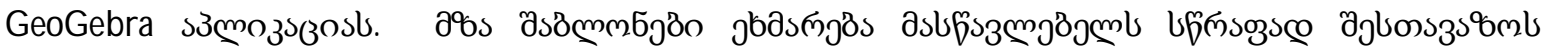

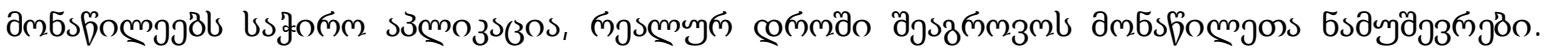

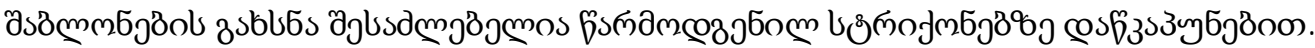

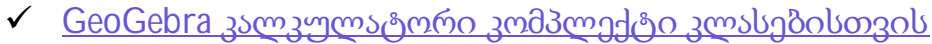

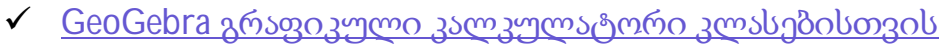 \\ $\checkmark$ GeoGebra zәмадj mos zmslujöolonzols \\ $\checkmark$ GeoGebra Classic zmsligönlonzols

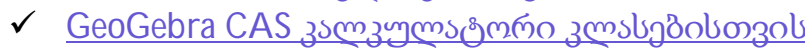

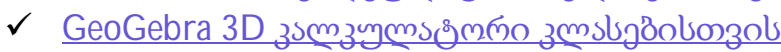

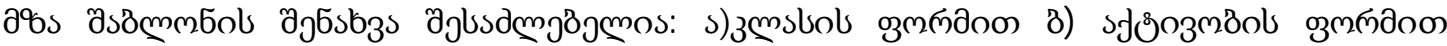

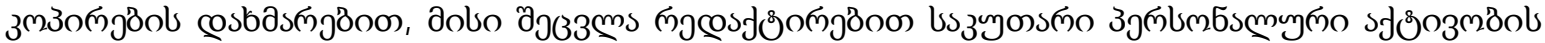

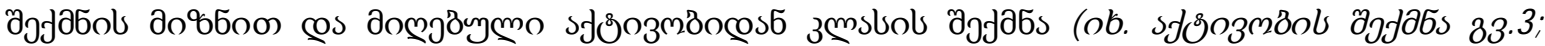

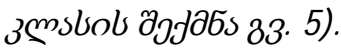

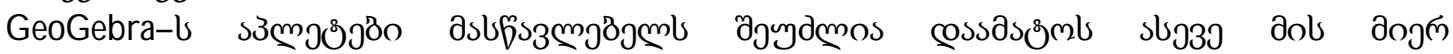

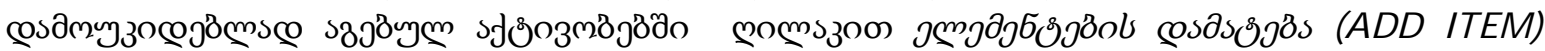

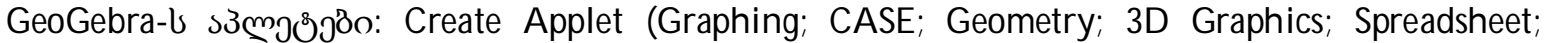
Probability; N otes.

\section{GeoGebra Class-ou gjła6s fozбocos6 GeoGebra Book}

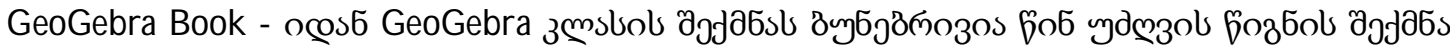

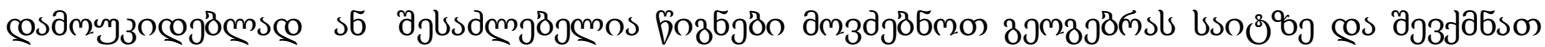

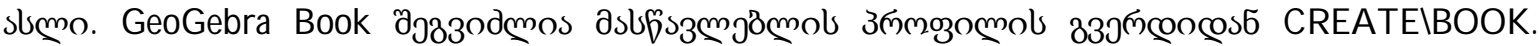

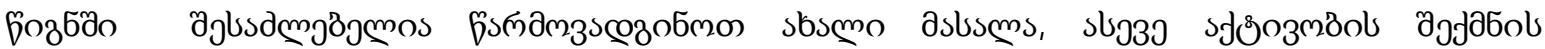




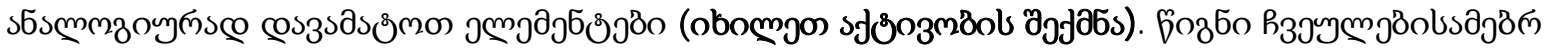

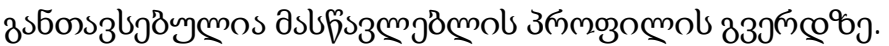

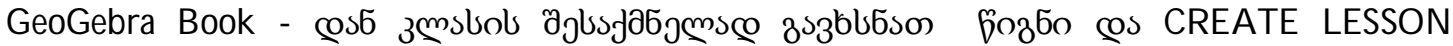

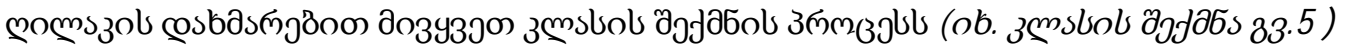

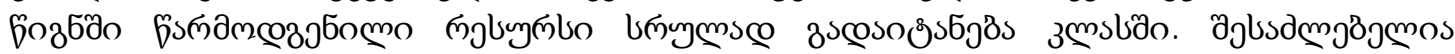

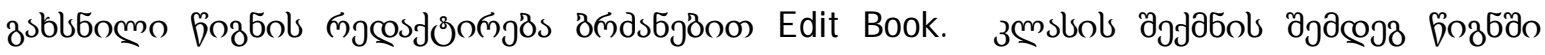

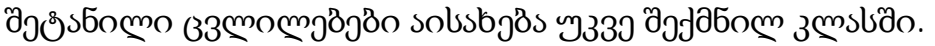

\section{cost $336 s$}

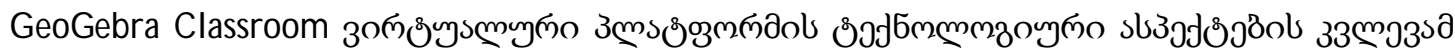

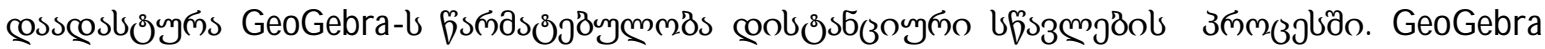

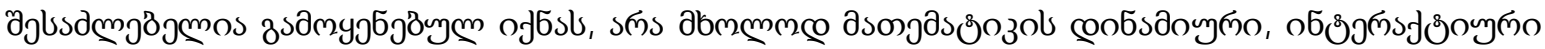

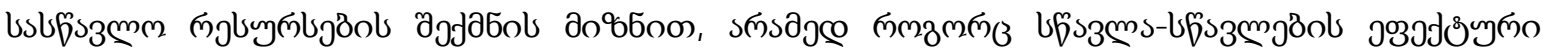

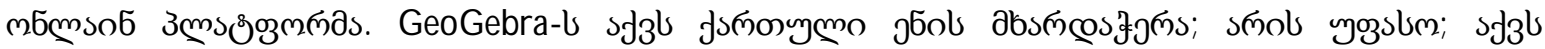

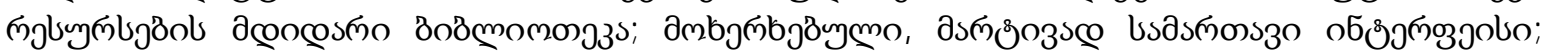

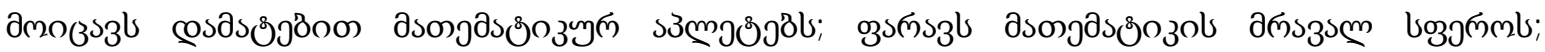

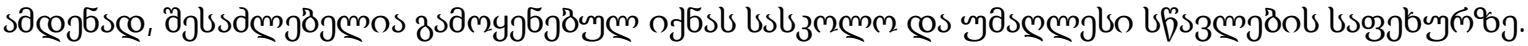

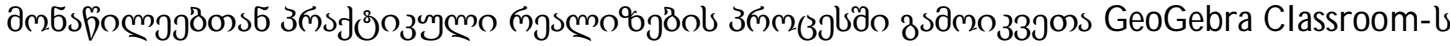

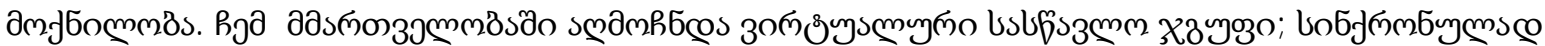

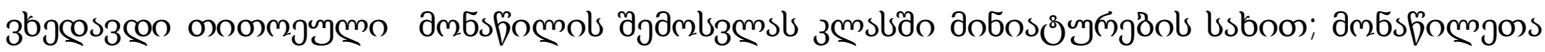

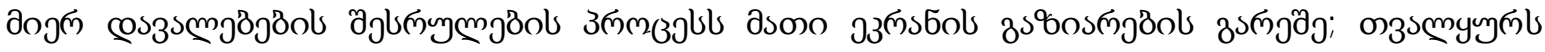

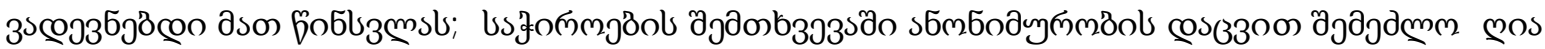

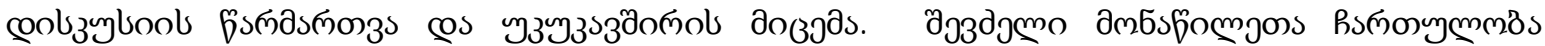

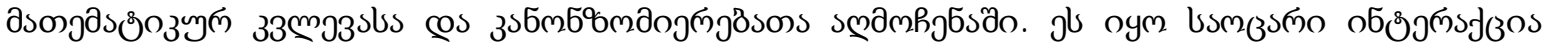

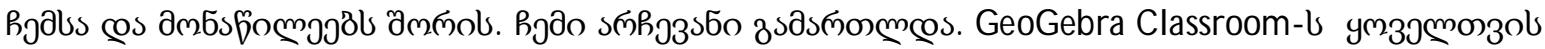

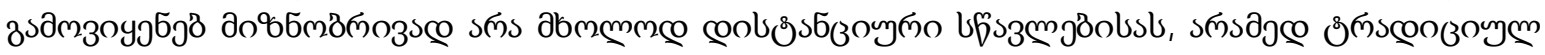

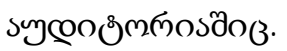

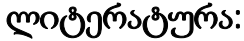

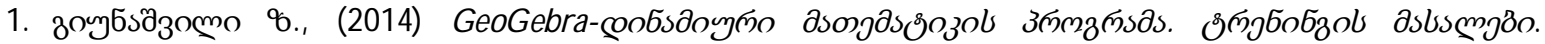

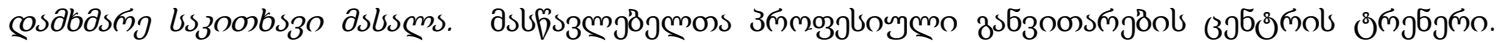
ö̊nmolo.

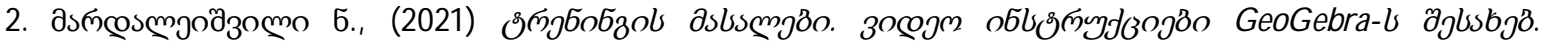

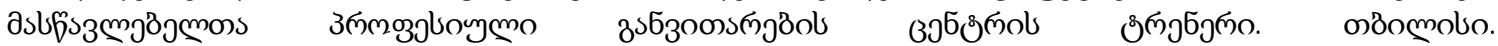

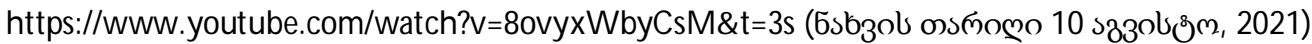

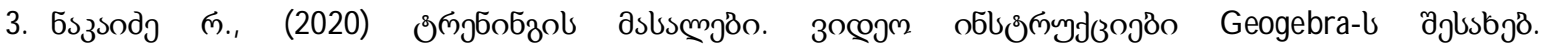

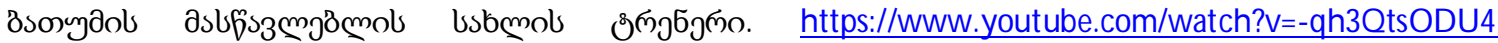

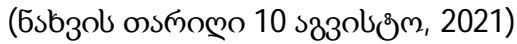

4. https://www.geogebra.org/ GeoGebra for Teaching and Learning Math GeoGebra - b mozozosmymo bsoత̊o. 


\title{
Technological Aspects of Distance Learning in Geogebra Classroom-Math Environment
}

\author{
Zakariashvili Mariam \\ Iakob Gogebashvili State University, Telavi
}

\begin{abstract}
The article discusses the technological aspects of distance learning in the GeoGebra Classroom virtual math platform environment, based on a practical model of using the GeoGebra Classroom. In particular, the main activities of Geogebra Classroom are presented in the following order: Activities - find / create an activity; GeoGebra class - create a GeoGebra class; Admission of students to the GoeGebra class; Appointment of a co-teacher; Monitoring student progress; GeoGebra classrooms in a distance learning environment; GeoGebra Applets (Graphing; CASE; Geometry; 3D Graphics; Spreadsheet; Probability; Notes) for GeoGebra Classroom; Creating a GeoGebra class from a GeoGebra book.

Every activity in the work is explained and performed in practice, which is confirmed by the corresponding illustrations actually carried out in the GeoGebra classroom environment. The algorithm for carrying out each activity is explained in detail and analyzed from a technological point of view and the methodological value of a separate step is shown. The discussion of the individual activities is accompanied by the proper definitions and purposes of the GeoGebra Classroom environment interface.

The article provides the recommendations for the necessary measures before starting the practical work in the GeoGebra Classroom,such as: Registration on the official GeoGebra website https://www.geogebra.org/; Download the GeoGebra Classic installation package and install it on the local computer; Have knowledge of using online and installed applications of GeoGebra Classic to create a resource.

The main activities of the GeoGebra classroom in the work are carried out on the example of discussing a practical model, parallel lines, with virtual groups of students. The following steps are shown sequentially: Creating resources in local and online GeoGebra applications; Creation of activities and their enrichment with additional elements of educational goals (Text; GeoGebra Applet; Notes; Question: Open question, Multiple choice; Video; Picture; PDF file Web); Creating a GeoGebra class and engaging participants online in real time; interactive communication in the GeoGebra classroom with the joint participation of the teacher and sstudents, which does not require sharing of screens by students; Tracking students' progress in the educational process, conducting anonymous discussions and providing feedback.

The article analyzes and confirms the success of using GeoGebra Classroom in teaching mathematics both in schools and in higher educational institutions.
\end{abstract}

Keywords: GeoGebra.org-Math; GeoGebra Classic App; GeoGebra Classroom - interactive online environment; GeoGebra Active, Book, Class. 